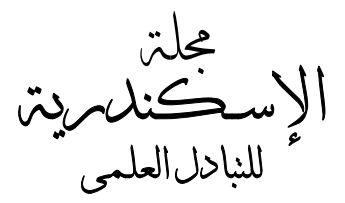

$r \cdot 10$

يوليو -سبتمبر

مجلد 7

الجدارة لافتصالية لانتاج اللحوم الحمراءف الاراضي الجية (درلسة حالة فمظاقة النوباربة)

عمرو عبد الحميد رفعت

الحيولنية(1) التبلغ حوالل 1,VV في حن تبلغ حوالل

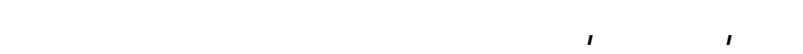

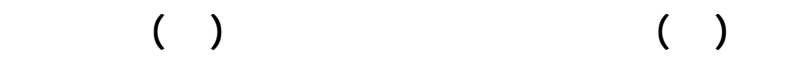

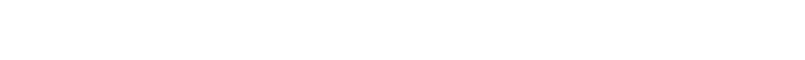

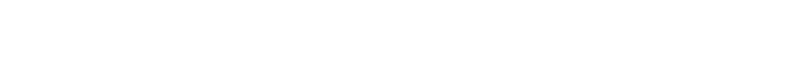
تنتساوى قيمة لنتلجينها الحية مع تكلفة فرصنها البيلة.

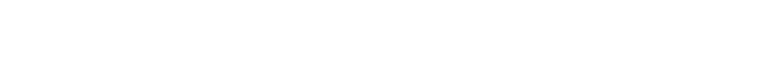

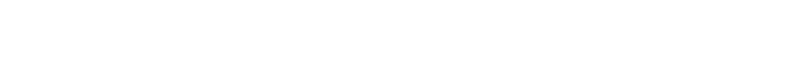

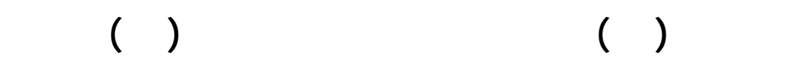

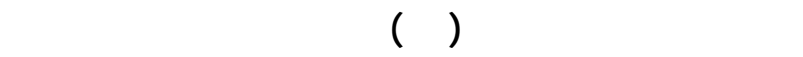

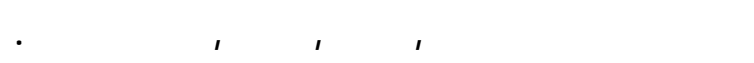

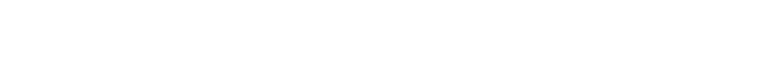
فرصنها البيلة اللعرف عل الجدالرة الفتصاية في السنخدلم

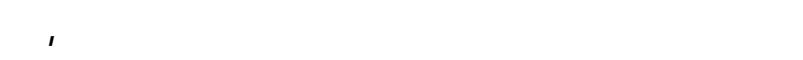

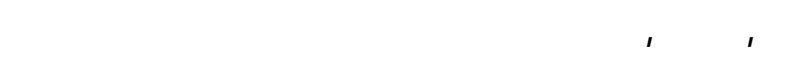

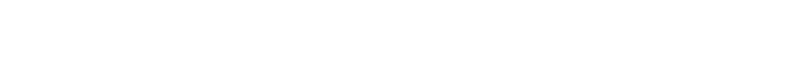

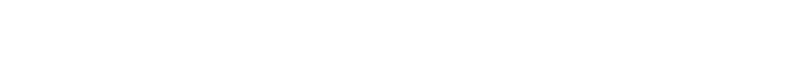

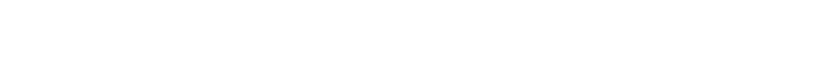

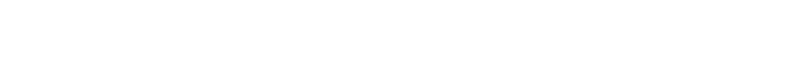
الخضراء حق تنتساوى قيمة لنتلجينها الحية مع تكلة فرصنتها البعيلة. وصففة علمة فتد التضح فوق مزالرع الفئة الحيازبة الثانية

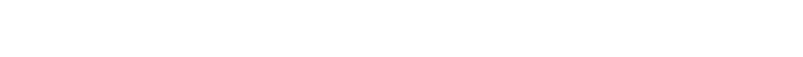

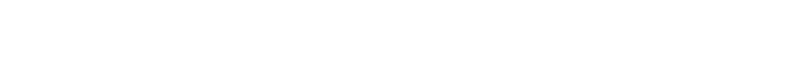

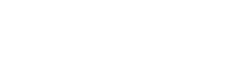

لمتهف هذا البحث قدير الدلات الانتلجية الحوم الحمراء في مزالرع الانتاج الحيولف باليم النواربة بلاراضي الجيية

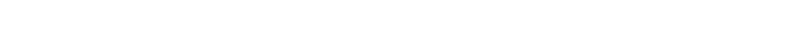

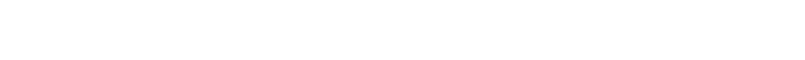

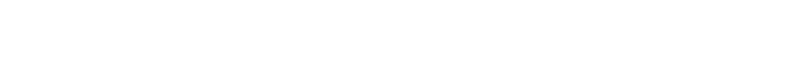

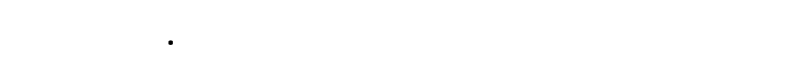

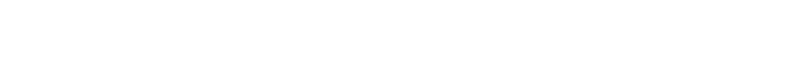

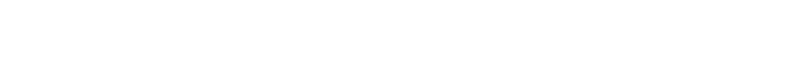
مزرعة وم قسيمها الي فئنين الاول تشطل المزالرع الت تضم الفماء

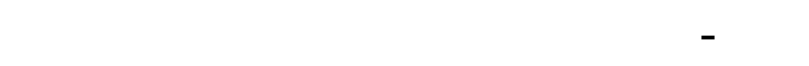
الزالرع الث تضم كثر من 0 وحدلت حيولنية وعنها

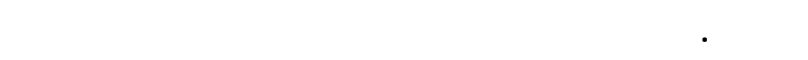

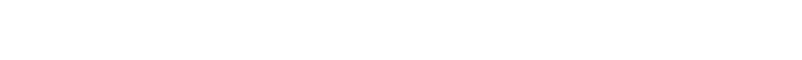
واللوغاربتمية والنصف لوغاربتمية والتربيعية وذك المئق عينة المرلسة.

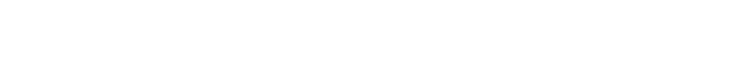
النتاج اللحوم الحمراء بمزالع الفئة الاول هـ عدد الوحدات

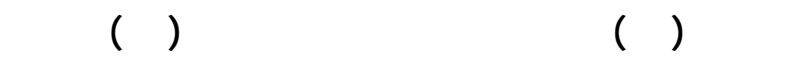
لاعلاف الخضراء باللان(س) وقد بلفت المرونة الانتلجية لهنه

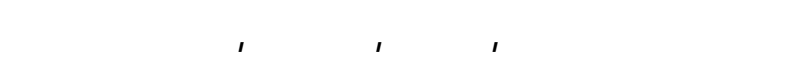
وفقدير نسبة فيمة الانتاجية الحية لهنه الموارد الل تكالفة فرصنها البيلة التعرف عل الجدارة الفتصاسية في لستخدالم الموارسسالفة النكر الضّح لن هذه النسبة لمتغير عدد الوحدلت

1أستاذ بلمث مساعد - قنم الدرلسلت الاقتصادية - مركز بحوث الصحراء

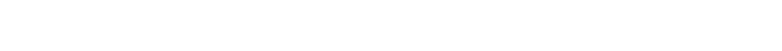


وهنا تجدر المثارة المي ان زياة التتجية الوحة الحيولنية

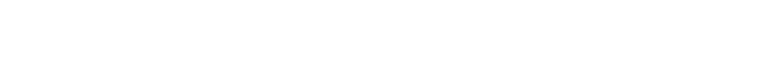
قصيرة نسبيا ولكن تولجه كقبت كثيرة من الصعب التغلب على غالبيتها خاصة والنها مرتبطة بالمزارع بالاراضي القديمة، وفي ضوء ذلك. يمكن القول بلن تنمية وظوير الثروة الحيوانية في جمهورية مصر العربية يمل في المرحلة الحالية والمسقبلية هدفا قوميا مرتبط بالاراضي الجدية خاصة

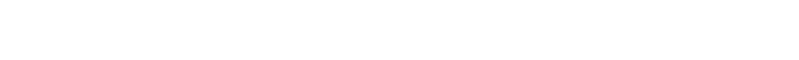

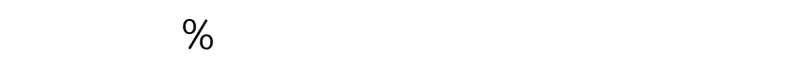
المنزرعة في مصر،هذا بالاضافة الي الدور الذي تلعبه

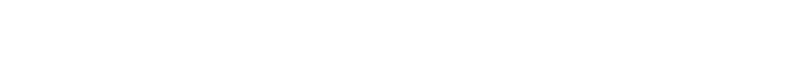

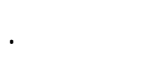

\section{له dx الهث}

يهوف البهث الي ققدير الدلات الانتلجية للحوم الحمراء

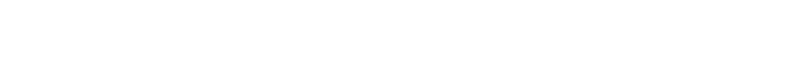

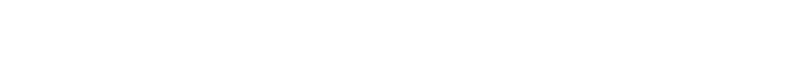

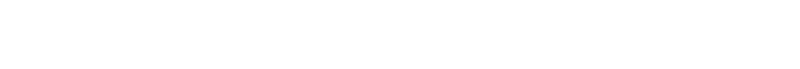
الحمراء بهزه المزراع وذلك للتعرف على مدى الكفاءة الماته الاقتصادية المزراعية المتلحة بمزراع النتاج اللحوم الحمراء

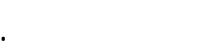

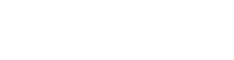

اعتمد البهث على بيانت اولية م الحصول عليها عن

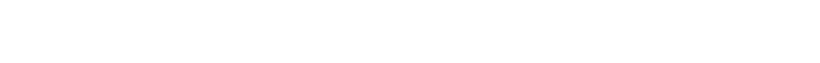

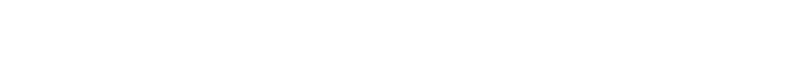

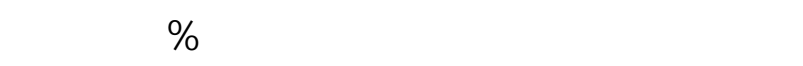
مزراع إنتاج اللحوم بالأقليم. وقد قم تقسيم العينة الي فئنين

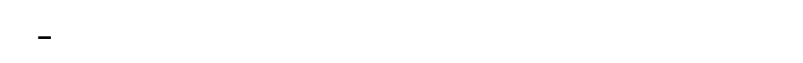

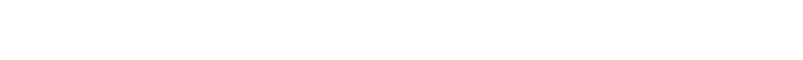

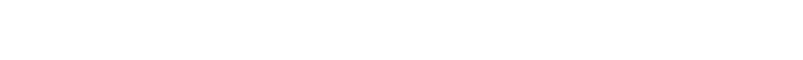
فئة منهما على حلة حيث تبين وجود فروق معنوية بين فئني

\section{المزرعية المتالة، وهو الامر الني يهف الزياد الانتاجية

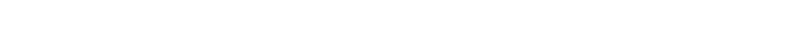 الحيولنية المستخمة بزيبادة اللسعة المزرعية الحيولنية. اللقمد}

يعتبر تحقيق الامن الغذائي وتوفير الحتيلجلت الغذائية

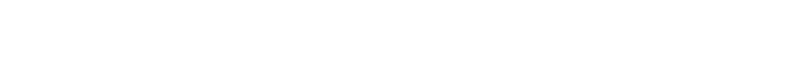
لتقققها كما ان النهوض بالمستوى الصهى اللسكان عن

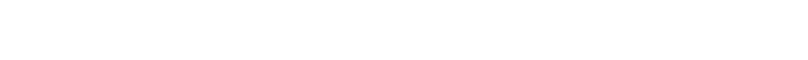

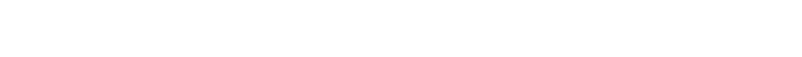
الغذاء اهم من كميته خاصه وان هناك علاقة وطية بين التخنية الجية والصجة الجسية والذهنية الافرادويملا فص البروتين الحيولف المشكلة المسلسية في تغذنية الفرد الفرد

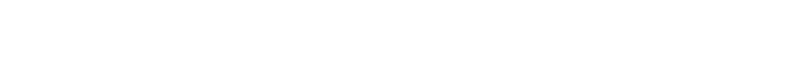
البروتين الحيولف ·لمجرلم في حين ما يحصل علية حالياً

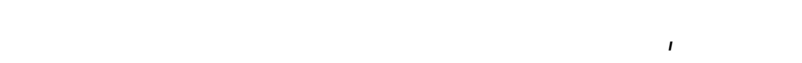
قص كبير بين الحتيلجلت الضرورية من البروتين الحيولف وبين المتاح منها للمستهلاك اليومه. ولاثك الن النيات هذا الموضع للسابق الاشارة الية هو نتاج فجوة غذائية تتمل في عجز الطاقة الانتلجية عن تلبية الاجتيلجلت المستهلكية المالية

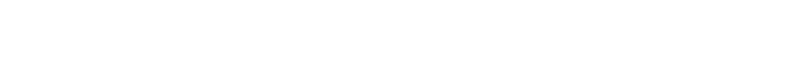
التغلب على هذه الفجوة. وظرا إلي ان لحمالات التوسع الأفقي لإنتاج البروتين الحيوالف بالأراضي القيمة في مصر تعتبر محدوة نسبياً

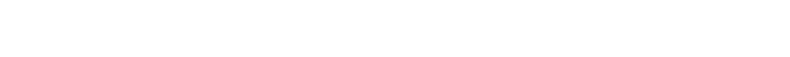

تاك الاحتيلجت تتنحصر في كل من: | -التوسع الرئسي بزياة التتجية الوحة الحيولية كن طريق: أ - التباع القنيت الحيية في الانتاج ب - التعسين الوراثي r - تنمية وطوير الثروة الجيوانية بالاراضي الجدية. 
لنموذج القدير لمعالم العلاقلت الاقتصاية القيلسية، وقد مُ

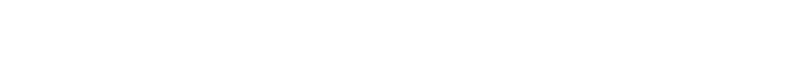

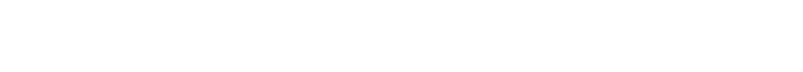
والتربيعية وذلك لفئتي عينة الدرلسة.

\section{النتائج البحثية}

الالفة الإنتلجية للحوم الحمراءف جمهوربة مصر العربية أولاً: قالور لالفة الإنتلجيةمن الموم الحمراء:

بدرلسة ظور الطاقة الإنتلجية للحوم الحم _راء خ لله

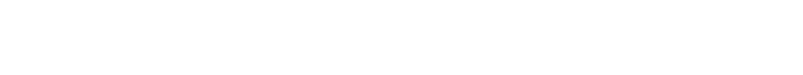

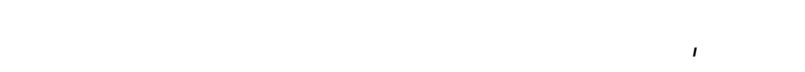

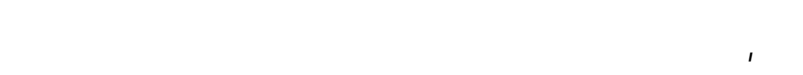

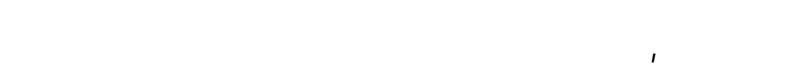

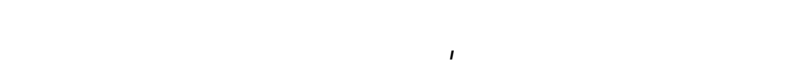

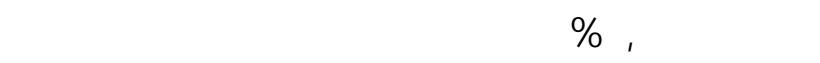

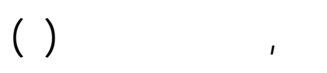

\section{لنهم محددت إنتاج اللحوف ف جمهوربة مصر العربية} ألولاً ظلور الوحدك الحيولنية ف جمهوربة مصر العربية:

\section{I - طلور الوحدل الحيولنية من الأقار}

بدرلسة طور الوحدات الحيوانية من الأبقار خلل الفترة 

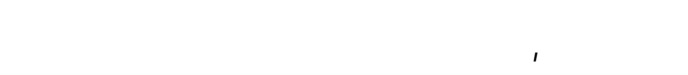

عينة الدرلسة من حيث متوسط الانتلجية للوحة الحيوانية

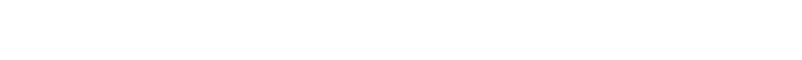
بلغت نسبة (ف) المحسوية حواله r, Y ع وهي معنوية عند

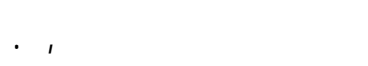

\section{لالسلوب البحثي}

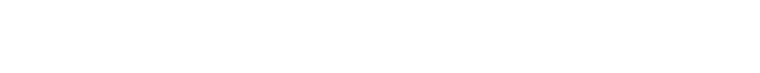

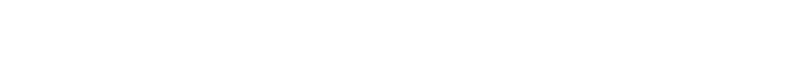

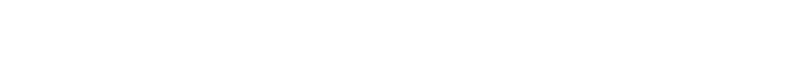

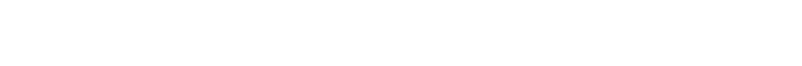

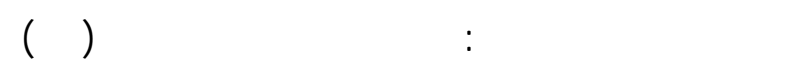

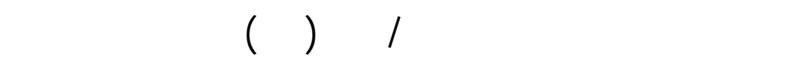

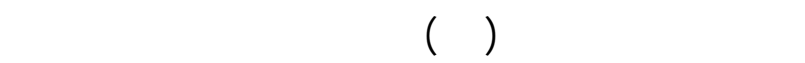

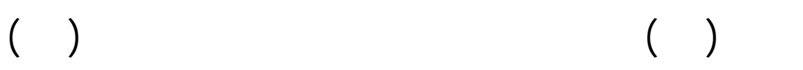

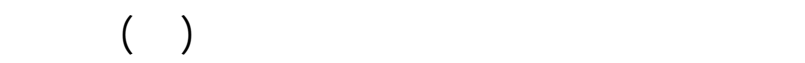

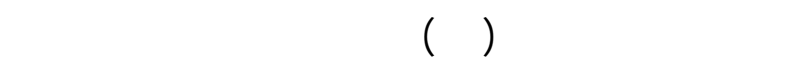

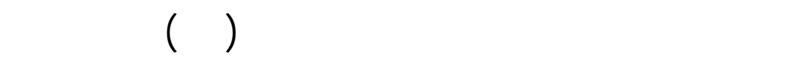
البيانات الوارة بالجدول رق م (1)متوس ط المتغي -رات

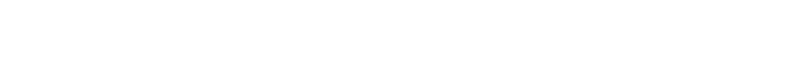

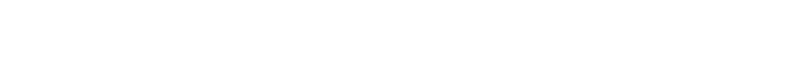
الجيية. وق للد تخدلم تحلي لل الانح عار المرحل قى المتع عدد Step- Wise Multi Regression جلط ال.متوطط المتغيرات المسقلة موضع الرالسة في مزراع الفئة الحيازبة الالل والثلنية بمزالر العينة البحثية في

\begin{tabular}{|c|c|c|c|}
\hline \multicolumn{2}{|c|}{ Iالمتوطa } & \multirow[t]{2}{*}{ الوحة } & \multirow[t]{2}{*}{ المتخيرت } \\
\hline الفئة الثلنية & الهئة الول & & \\
\hline $\mathrm{V}, 0$ & $r, \Lambda$ & وحلة حيوانية & عددالوحدات الحيولنية (u) \\
\hline 110 & 07 & رجل/يوم & العمالة|لمزرعية (سنr) \\
\hline 17. & $\mathrm{~V} \cdot$ & جنية & الادوية البطرية(سr) \\
\hline $0, \varepsilon$ & $r, 0$ & طن & كمية الاعلاف المركزة (سع) \\
\hline 11 & $\Lambda, \mathrm{VO}$ & طن & كميةالاعلاف الخضراء (ou) \\
\hline 7,0 & $r, I \varepsilon$ & طن & كميةالاعلف المالئة الجلفة (س)ر) \\
\hline r & rq & بالسنين & عمر المربي (سv) \\
\hline $1 \mu$ & 1 . & عدد للسنوات الت قضاها في التعليم & مستوى تعليم المربي (uم) \\
\hline
\end{tabular}




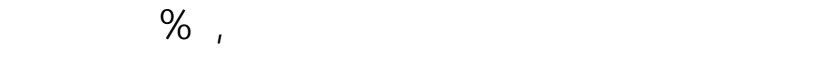
معنويتها عند مستوى معنوية 1 •, . r - طلور الوحدات الحيولنية من الأغنله:

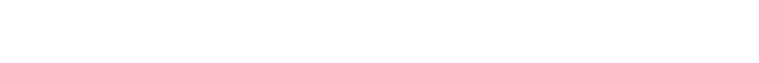

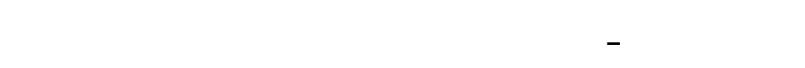

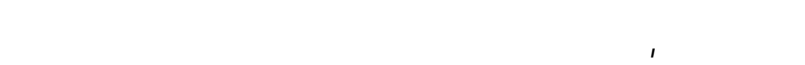

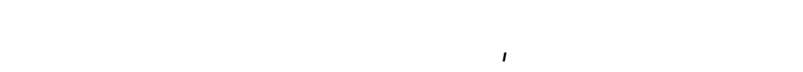

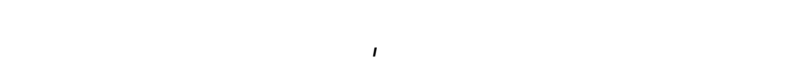

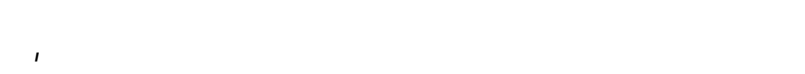

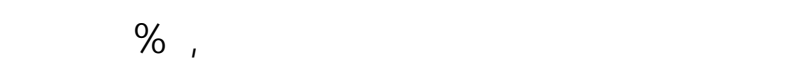

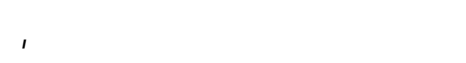

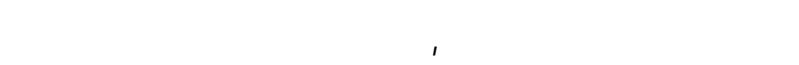

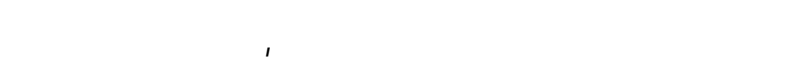

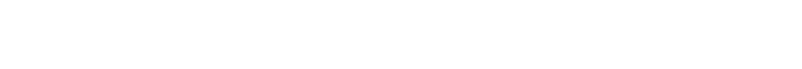

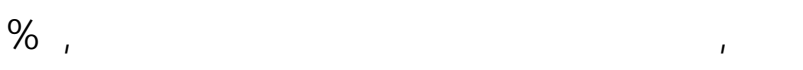
وقد ثبت معنويتها عند مستوى معنوية ال ال, r - ظلور الوحدات الحيولنية من الجالمون:

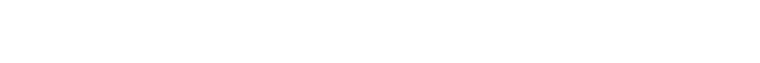

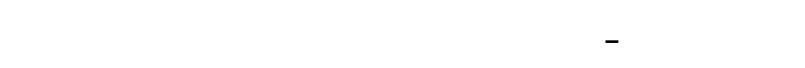

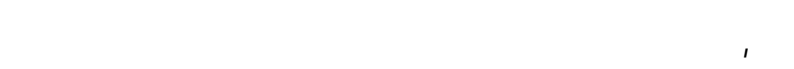

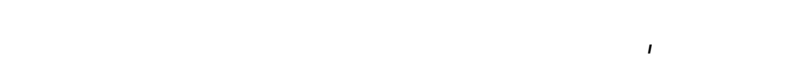

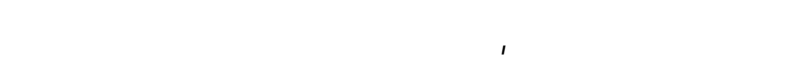

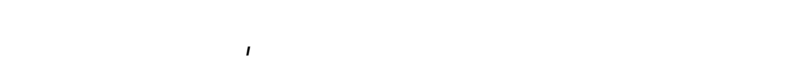

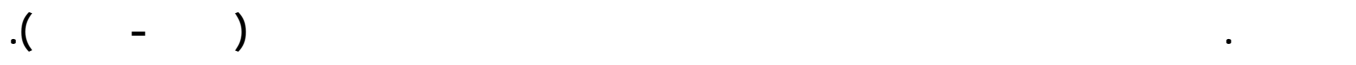

\begin{tabular}{|c|c|c|c|c|c|c|}
\hline الإجمالل & الجمل & الماعز & الأغنله & الجالموس & لالقار & اللسنة \\
\hline $07 r, 101$ & $Y V, V \Lambda$ & $\mu \cdot, 90$ & $7 \varepsilon, \mu q$ & $179, \cdot{ }^{\mu}$ & $r V \cdot, \cdot 1$ & 1991 \\
\hline OVE,rq & $\langle\Lambda, 7\rceil$ & ${ }^{\mu}, \mathrm{T}$. & $7 \varepsilon, 97$ & $\mid V Y, V I$ & rVo,Or & 1995 \\
\hline 7Vץ,orE & $\Upsilon \Lambda, 19$ & $r 1,9 V$ & $7 \pi, \varepsilon\rceil$ & IV9, V9 & YMI,IT & $199 \mu$ \\
\hline$V 70,771$ & 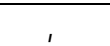 & ${ }^{\mu V}, V_{0}$ & $09, Y 7$ & 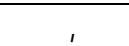 & $r \wedge V, \varepsilon$. & $199 \varepsilon$ \\
\hline$\left.V^{\mu} \Lambda, \Lambda \cdot\right\rceil$ & $\mathrm{V \Lambda , \varepsilon \varepsilon}$ & $\mu q, \Lambda \Lambda$ & $\mathrm{V \varepsilon}, \mathrm{V} \mathrm{T}$ & $r 7 \cdot, \Lambda \mu$ & $r \wedge \varepsilon, q \cdot$ & 1990 \\
\hline$\Lambda \varepsilon 1, \wedge 09$ & $\varepsilon q, \wedge \varepsilon$ & $\mu \Lambda, 70$ & $00^{4}, 19$ & ro7,01 & $\mu \varepsilon \psi,\rceil \Lambda$ & 1997 \\
\hline १દ१,૦૧દ & $0 \varepsilon, q$. & $\varepsilon 1, V^{\mu}$ & $71, \mathrm{YV}$ & ${ }^{\mu} \Lambda \Lambda, \mu \mid$ & $\varepsilon \cdot \mu, \mu q$ & $199 \mathrm{~V}$ \\
\hline $7 V \cdot, \cdot \cdot \mu$ & $\Lambda, \cdot \cdot$ & $0 \cdot, \cdot \cdot$ & $v_{1}, \cdots$ & $r \wedge \mu, \cdot 1$ & rOA, & 1991 \\
\hline $7 \Lambda \Lambda, 1 \cdot 7$ & $\mathrm{~V}, \mathrm{\Lambda} \cdot$ & $\varepsilon q, q$. & $v \cdot, q \cdot$ & $r \wedge 7,1$. & $r \vee r, \varepsilon$. & 1999 \\
\hline$V \cdot r, \mu \wedge \varepsilon$ & $\Lambda, \cdot \cdot$ & $01, \varepsilon$. & $V r, 99$ & $r \Lambda \Lambda, \cdot \cdot$ & rAY,. & $r \ldots$ \\
\hline $791,7 \cdot 7$ & $V, V$. & $0 r, \mu$. & $\begin{array}{ll}V \varepsilon, \Lambda I \\
\end{array}$ & 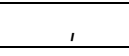 & YYY,19 & $r \cdot 1$ \\
\hline$\Lambda \mathrm{V}, \Lambda \cdot V$ & $V, \varepsilon$ & $\mathrm{Or}^{\top}, \Lambda$. & $\Lambda r, \mathrm{~V}$. & ${ }^{\mu} \Psi, 0$ & $\mu \mu, \varepsilon$. & $r \cdot r$ \\
\hline$\Lambda \cdot 0,11 \Gamma$ & $V, q$. & $07,9$. & $\Lambda \mathrm{\Lambda}, \mathrm{V})$ & ${ }^{\mu}{ }^{\prime}$ & r५q,\%. & $r \cdot \mu$ \\
\hline 110,199 & $\mathrm{~V}, \mathrm{O}$. & $07,9$. & $\Lambda \cdot, r$. & $\mu^{\mu} \cdot, 1$. & $\mu \varepsilon \cdot, 0$. & $r \cdot \varepsilon$ \\
\hline$\Lambda 0 \mathrm{\Lambda}, \Lambda \mathrm{\top}$ & $\Lambda, Y\urcorner$ & $0 \varepsilon, \mathrm{V} \varepsilon$ & $\left.\Lambda \mu^{\mu}, \varepsilon\right\rceil$ & ror, 70 & ror,vo & $r \cdot 0$ \\
\hline$\Lambda V 7$ & $\Lambda, 7$ & $00, \cdots$ & 10,0 & $r 7 \cdot, r$. & $\mu 77, \mathrm{~V}$. & $r \cdot .7$ \\
\hline $91 \varepsilon, \mu \cdot \varepsilon$ & $\varepsilon, q$. & $7 \cdot, \Lambda \cdot$ & $\Lambda 0, \Lambda$. & $\mu 79,7$. & rqu,r. & $r \cdot v$ \\
\hline १०८,ह११ & 7,1 . & $71, \mu$. & $\Lambda 7, r$. & rVo, & $\varepsilon \Upsilon q, q$. & $r \cdot \Lambda$ \\
\hline $1 \cdot 1 \cdot, 9 \mathrm{~V}^{\mu}$ & $V, 90$ & $09, \mathrm{r} 7$ & $\Lambda 0,1 \Lambda$ & $\varepsilon 11,9 \pi$ & $\varepsilon \varepsilon 7, \overline{T V}$ & $r \cdot q$ \\
\hline $99 \%, \cdot \cdots 1$ & $\Lambda, 0 \mathrm{~V}$ & $0 r, 77$ & Vo,IT & 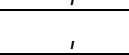 & $\varepsilon O V, \mu$. & r.l. \\
\hline $9 \Lambda \Lambda, \cdot 0 r$ & $1 \cdot, 71$ & Or, Or & $V^{\mu}, 7 \varepsilon$ & $\mu 90, \Lambda$. & $\varepsilon 0 \varepsilon, \varepsilon \Lambda$ & $r .11$ \\
\hline $9 \wedge 9,0 \cdot 9$ & $1 \cdot, 9 \mathrm{~V}$ & $0 \varepsilon, 0$. & $\mathrm{VE}, 0{ }^{\mu}$ & $r \wedge \varepsilon, q \mu$ & $\varepsilon\urcorner \varepsilon, 0 \Lambda$ & $r \cdot \| r$ \\
\hline$\Lambda \cdot \Lambda, 1 \cdot 91$ & $19, \cdot v$ & $\varepsilon \Lambda, \varepsilon \Lambda$ & $V \varepsilon, \cdot 0$ & 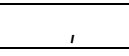 & $\mu \varepsilon 0,70$ & المتوبb \\
\hline
\end{tabular}
المصدر: الجهاز المركزى للتعبئة العلمة والإحصاء ، الدرلسة للسنوية لظور حرركة الإنتاج والنجارة الخارجية والمتاح من الإستهلاك من للسلع 


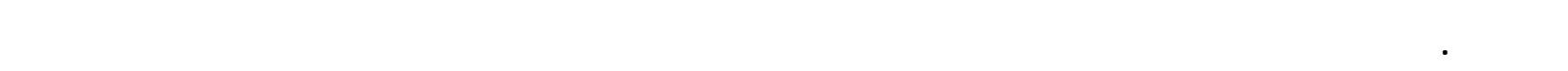

\begin{tabular}{|c|c|c|c|c|c|}
\hline الجمل & الماعز & لأغنله & الجاموس & لأققار & لالسنة \\
\hline$r \cdot \Lambda$ & $\varepsilon 79 r$ & $\varepsilon r V$. & rorv & rorv & 1991 \\
\hline rIV & $0 . r$. & $\varepsilon \mu \backslash \Lambda$ & ro\&A & $\mu 71 r$ & 1994 \\
\hline r r & OrVr & EOH. & rov. & $\mu\urcorner \Lambda \Lambda$ & $199 \%$ \\
\hline rq. & $\mu 191$ & $\varepsilon 901$ & rI19 & rVYA & $199 \varepsilon$ \\
\hline ארץ & 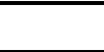 & 0771 & rYعO & $r V \cdot \varepsilon$ & 1990 \\
\hline$r 00$ & нгкq & EOH $\Lambda$ & 正 & זרזץ & 1997 \\
\hline$r \Lambda$. & rE77 & OrEA & roVE & 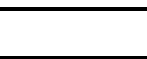 & $199 V$ \\
\hline IEr & זrרI & Eror & ए। & & 1991 \\
\hline $1 \mu \varepsilon$ & $\mu \mu \cdot \Lambda$ & $\varepsilon \mu q 1$ & . & $\mu \varepsilon \mid \Lambda$ & 1999 \\
\hline$|\varepsilon|$ & rEro & हع 79 & ruvq & ror. & $r \cdots$ \\
\hline $1 \mu \varepsilon$ & ץ६qV & $\varepsilon 7 V I$ & rorr & $\mu \wedge \cdot 1$ & $r \cdot r$ \\
\hline IrV & rOAr & 01.0 & rVIV & $\varepsilon \cdot \Lambda r$ & $r \cdot r$ \\
\hline$|\mu|$ & एAII & ह१щ१ & rVVV & EYYV & $r \cdots \mu$ \\
\hline 149 & ऍ^Vq & $0 \cdot \varepsilon \mu$ & 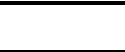 & $\varepsilon \mu\urcorner 9$ & $r \cdot \varepsilon \varepsilon$ \\
\hline $1 \varepsilon r$ & $\mu \Lambda \cdot \mu$ & ORHY & $\mu \wedge \wedge 0$ & $\varepsilon \varepsilon \wedge 0$ & $r \cdots 0$ \\
\hline$I \varepsilon \Lambda$ & ґАVV & Or^o & 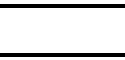 & $\varepsilon 71$. & $r \cdot r$ \\
\hline$\Lambda \varepsilon$ & $\varepsilon r \|$ & OहTV & $\varepsilon 1 \cdot 0$ & 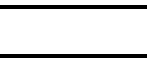 & $r \cdots V$ \\
\hline $1 \cdot V$ & $\varepsilon \varepsilon V^{\mu}$ & Oع91 & $\varepsilon \cdot O^{\mu}$ & $0 . \mathrm{r \mu}^{\mu}$ & $r \cdots \Lambda$ \\
\hline $1 \mu V$ & $\varepsilon)^{\mu q}$ & 0094 & $\mu \wedge \mu q$ & EOro & $r \cdot .9$ \\
\hline 111 & EIVO & $00 \mu$. & 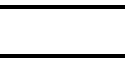 & $\varepsilon \vee r q$ & $r \cdot 1$. \\
\hline $1 \mu V$ & $\varepsilon Y O \Lambda$ & $0 \mu 70$ & $\mu q \Lambda \mu$ & $\varepsilon \vee \Lambda$. & $r .11$ \\
\hline $1 \varepsilon r$ & $\varepsilon^{\mu} \cdot 7$ & Oहץ. & $\varepsilon 170$ & દ૧દ૫ & $r \cdot 1 r$ \\
\hline IVT & r9r7 & $\varepsilon 999$ & $\mu \varepsilon \mu \mu$ & $\mu \wedge|7, \mu|$ & المتوطط \\
\hline
\end{tabular}

المصدر: ـ الجهاز المركزى للتعبئة العلمة والإحصاء ، الكتب الإحصائى للسنوى ، أعداد مقفرقة.

\section{0 - ظلور الوحدات الحيولنية من الجمل:}

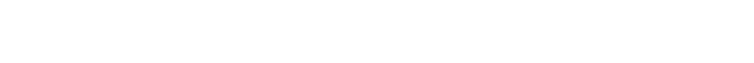

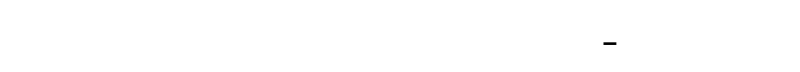

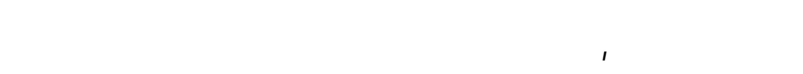

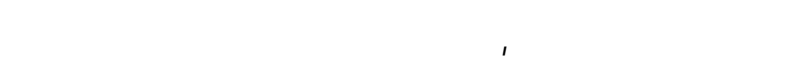

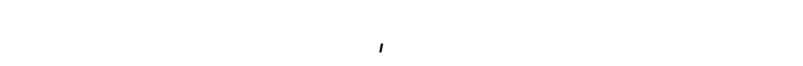

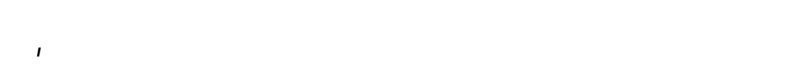

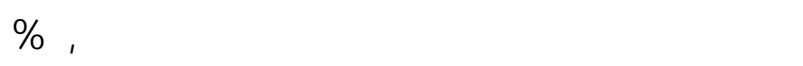
وقد ثبت معنويتها عند مستوى معنوية ا >, أ.

\section{ع - خلور الوحدات الحيولنية من الماعز:}

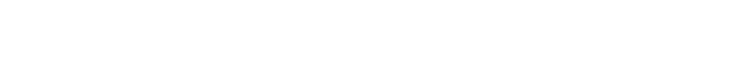

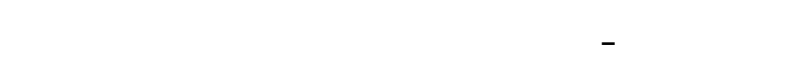

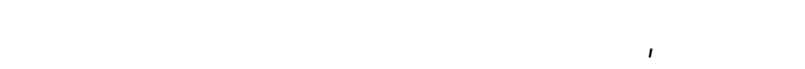

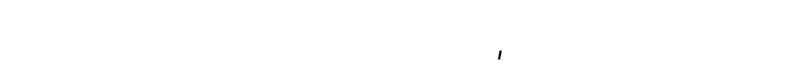

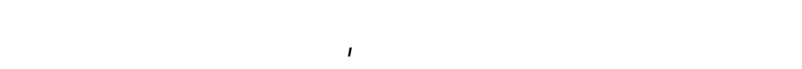

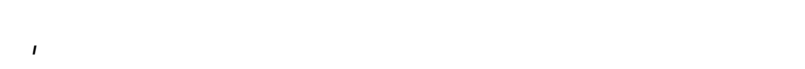

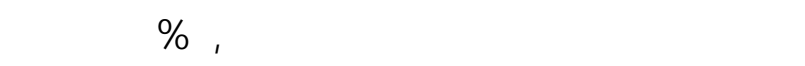
معنويتها عند مستوى معنوية ال . ,. 


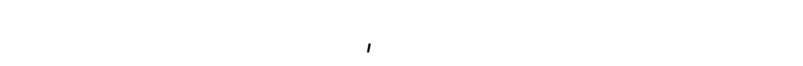
1 - ظلور إجمال الوحدل الحيولنية:

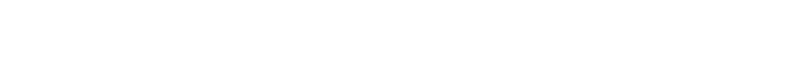

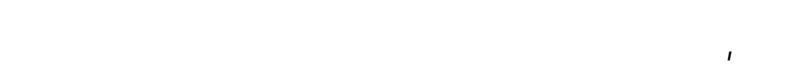

بدرلسة قطور إجمالى الوحدات الحيوانية خلل الفت ـرة

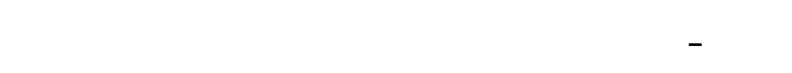

TrV

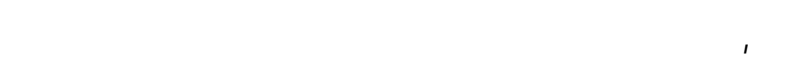

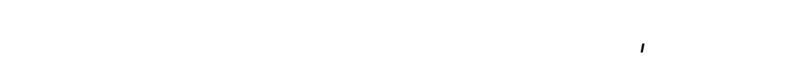

\begin{tabular}{|c|c|c|c|c|c|c|}
\hline لإجمال & الجمل & الماعز & لأغنلم & الجاموس & لأبقار & لللسنة \\
\hline $\mathrm{V} 7 \cdot \mathrm{V}, \mathrm{II}$ & $107, \Gamma \mu$ & YYA,EY & हหา,११ & " & rorv, I. & 1991 \\
\hline દEहભ,$\cdot \Lambda$ & $17 \pi, 0{ }^{\mu}$ & rol,El & $\varepsilon^{\mu} \mid, \Lambda$. & 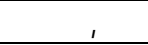 & malr,l. & 1994 \\
\hline $\mathrm{Vq \cdot \mu , \varepsilon V}$ & IV $\varepsilon, \cdots$ & $\mu \vee\urcorner, \cdot \varepsilon$ & $\varepsilon \mathrm{OH}^{\mu}, \cdot \cdot$ & "r, & $\mu\urcorner \wedge \vee, \Lambda$. & $199 \mu$ \\
\hline $7 \varepsilon \cdots, 0 \Lambda$ & $r I V, \Lambda \mu$ & $\Gamma{ }^{\mu} \mu, \Lambda \varepsilon$ & $\varepsilon 90, \cdot \Lambda$ & rVभ $7, I I$ & YVYV,VY & $199 \varepsilon$ \\
\hline $70 \wedge 0, \varepsilon q$ & rVI, AM & $r^{\mu} 7,01$ & $077,1 \mu$ & $r \Lambda \cdot\urcorner, \Lambda \varepsilon$ & $r V \cdot \varepsilon, \mid \varepsilon$ & 1990 \\
\hline$\left.\Lambda^{\mu \mu}\right\rceil, 00$ & 191,17 & $r r 7, V$. & $\varepsilon \mathrm{OH}^{\mu}, \Lambda \Lambda^{\mu}$ & $\varepsilon r \cdot \mu, r r$ & "ז, & 1997 \\
\hline$\Lambda V^{\mu} V^{\mu}, \mathrm{OH}^{\mu}$ & $r|\cdot, r|$ & rEr,, 1$]$ & OrE,,$\Lambda$ & EE $7 V, 1$. & 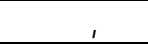 & $199 V$ \\
\hline 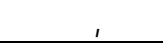 & $1 \cdot 7,11$ & $r Y \Lambda, Y V$ & $\varepsilon \mu 0,1 \wedge$ & 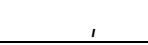 & PYI T,V. & 1991 \\
\hline$\Lambda^{\mu} \mathrm{O} \mathrm{I}, \mathrm{IV}$ & $1 \cdots, \Lambda r$ & rwו,OV & $\varepsilon \mu q, \cdot V$ & عI & 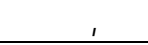 & 1999 \\
\hline$\wedge 0 \varepsilon 7,19$ & $1 \cdot 0,07$ & $\mu^{\mu q, V^{\mu}}$ & 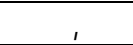 & $\varepsilon \Gamma \Upsilon \varepsilon, Y\rceil$ & rorq,Vr & $r \cdots$ \\
\hline $9 \cdot 17,1$. & $1 \cdots,{ }^{\mu} \Gamma$ & 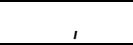 & $\varepsilon 7 V, I r$ & $\varepsilon \varepsilon \cdot r, \Lambda)$ & $\mu \Lambda \cdot I, \cdot V$ & $r \cdots 1$ \\
\hline $90 \wedge \mu, \Lambda\rceil$ & $90, \mu)$ & ro.,vר & $01 \cdot, \varepsilon 0$ & $\varepsilon\urcorner \varepsilon 0, \Lambda$. & $\varepsilon \cdot \Lambda 1,0 \mu^{\mu}$ & $r \cdots r$ \\
\hline $9 \Lambda \cdot V, 07$ & $9 \Lambda, \varepsilon \Lambda$ & rา7,VE & ह१ץ,१। & દVYI,દદ & हYYๆ,११ & $r \cdots \mu$ \\
\hline $1 \cdots \varepsilon \Lambda,{ }^{\mu r} Y$ & $9 \mathrm{~V}, \mathrm{Ir}$ & rVI,0E & $0 \cdot \varepsilon, r \Lambda$ & $\varepsilon \Lambda \cdot\rceil, \varepsilon \mu$ & $\varepsilon \mu\urcorner \Lambda, १ ०$ & $r \cdots \varepsilon$ \\
\hline I - $\mu_{\mu \nu, Y \mu}$ & $1 \cdot 7, \Lambda r$ & $r 77,1 \wedge$ & OrH,Y. & $\varepsilon \wedge 07,{ }^{\mu} \bigvee$ & $\varepsilon \varepsilon \wedge \varepsilon, 77$ & $r \cdots o$ \\
\hline I. 1 عOr, ${ }^{\mu} \mathrm{V}$ & $111,1$. & rVI,El & $O \mu \wedge, 0 \varepsilon$ & દ૧૫ા,૦ & $\varepsilon\urcorner \cdot 9, \vee \wedge$ & $r \cdots 7$ \\
\hline $1.97 \Lambda, 1 \mu$ & $7 r, 97$ & rqE,VO & $0 \varepsilon 7, \mathrm{~V} 0$ & $01 \mu 1, \cdot 1$ & ह१" & $r \cdots V$ \\
\hline $11 \cdot \mu^{\mu} \Upsilon, \varepsilon 0$ & $\Lambda \cdot, \mathrm{OH}^{\mu}$ & $\mu$ & 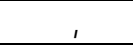 & $0 \cdot 70, \Lambda 1$ & $0 . \mu^{\mu}, 17$ & $r \cdots \Lambda$ \\
\hline $1 \cdot r V 0,9$ & $1 \cdot r, \Lambda \mu$ & 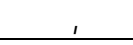 & 009,17 & $\varepsilon \vee १ \bigwedge, \varepsilon$. & દorદ,१૦ & $r \cdots q$ \\
\hline $1 \cdot \varepsilon r q, 70$ & $\Lambda r, q \mu$ & rqr,ro & $00 Y, 90$ & 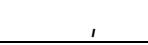 & EVYA,VY & $r \cdot 1$. \\
\hline $1.790,91$ & $1 \cdot r, v \cdot$ & $r 9 \Lambda, \cdot V$ & Or 7,01 & દ૧マ^,१७ & 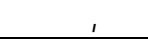 & $r .11$ \\
\hline $111 \cdot \mu, 11$ & $1 \cdot 7,10$ & $\mu \cdot 1, \varepsilon \varepsilon$ & OEr, 90 & $0 r \cdot 7,17$ & દ૧દ૫,દા & $r \cdot 1 r$ \\
\hline$q \cdot \Lambda \cdot, \cdot 0$ & $1 \mu \cdot, \mu\rceil$ & $r V^{\mu}, 00$ & 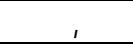 & $\varepsilon r \varepsilon V, \mu \varepsilon$ & $\mu \mu^{\mu} \cdot,{ }^{\mu}$ & المتوuط \\
\hline
\end{tabular}

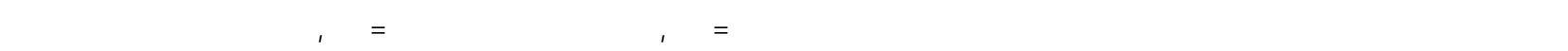

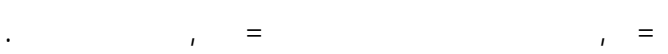

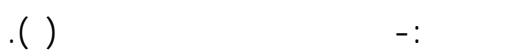

\begin{tabular}{|c|c|c|c|c|}
\hline ف & ני & ت & المعادلة & البيلن \\
\hline * $\urcorner \varepsilon, \vee \wedge \varepsilon$ & $\cdot, \mathrm{V}\urcorner \varepsilon$ & $\Lambda, \cdot \varepsilon q$ & ص & الأقبار \\
\hline *VE,IYV & $\cdot, \mathrm{V} \wedge \Lambda$ & $\Lambda, 71$ & ص & الجلموس \\
\hline $19, V \wedge$. & $\cdot, \varepsilon १ \bigvee$ & $\varepsilon, \varepsilon \varepsilon V$ & ص & الأغنله \\
\hline$\therefore \cdot \varepsilon 0$ & $\cdot, \cdot r$ & $\cdot, \mathrm{rII}$ & ص & الماءز \\
\hline${ }^{*} 19, \wedge 9 \%$ & $\cdot$, ह११ & $\varepsilon, \varepsilon\urcorner$. & ص & الجمل \\
\hline$* \| \cdot, \mu \cdot \varepsilon$ & $\cdot, \Lambda \varepsilon V$ & $1 \cdot, 0 \cdot \mu$ & & الإجمالم \\
\hline
\end{tabular}

• معنوى عند المستوى الإحتماله I ·,

المصدر: التحلبل الإحصائى للبيانت الوارةة بالجدول رقم (عائ (ع) 
الفسيرية في النموذج للسايق تغسر حواله 10\% من التبانن في لجماله الناتج المزرعي من اللحوه في مزارع الفئة

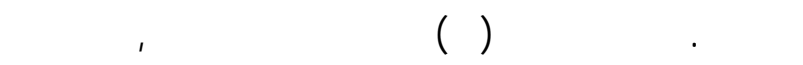
يعن معنوية تاثير المتغيرات الفنيرية في النموذج اللساق

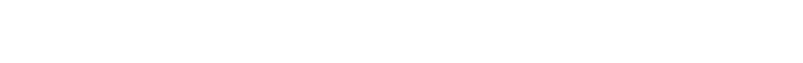
الحمراء في العينة البحثية عند المستوى الاحتماله ا ـ. . . وبلستعراض وتحليل المشقلت الاقتصاية من الدالة

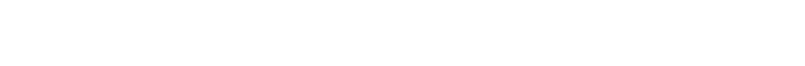

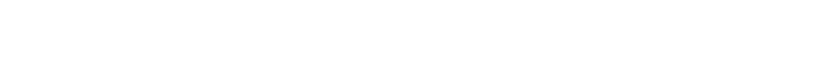
المركزة والاعلاف الخضراء بلغت على التواله حواله

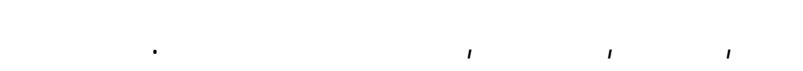

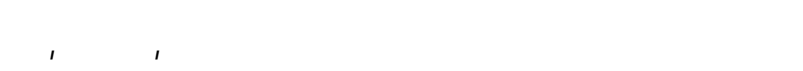
سך · , · طن على التواله. وبرسلب المرونة الانتلجية للمتغيرس(ا) اتضضح انها تبلغ

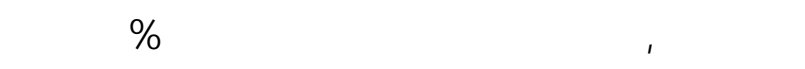

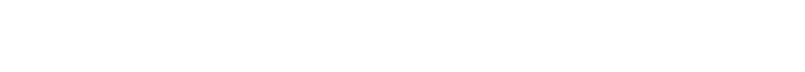

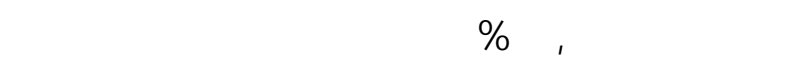
اللحو.وبالنسبة للمرونة الانتلجية للمتغير (سع) فقد بلغت

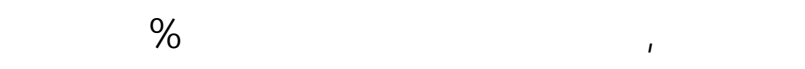
الاعلاف المركزة يترتب عليه تغيرًَ في فنس الاتجه مقدارو r,س\% في لجماله الناتج المزرعى من اللحوع.الما فيما يتعلق بالمرونة الانتلجية للمتغيرسم) فقد بلغت حواله اله

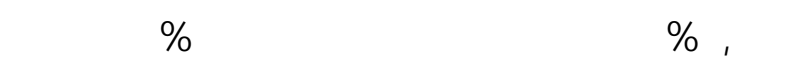
الاعلاف الخضراء يترتب عليه تغيرً في نفس الاتجه

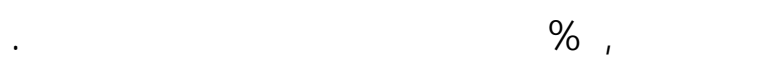
وبقيلس الكفاءة الاقتصادية للموارد المزرعية المستغلة

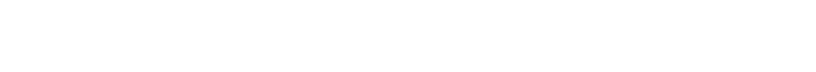

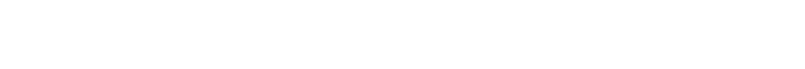
الموارد اله تكلفة فرصتها البيلة للتعرف على الجدارة الاقتصادية في لستخده المواردسالفة النكر
النتائج الخاصة بالمينة البحثية: -

الولا: - الدلات الانتلجية للحوم الحمراء في مزراع الفئة

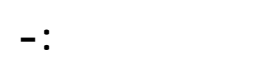

بلستخدلم تحليل الانحدار المرحله المتعدد لققدير الدالات

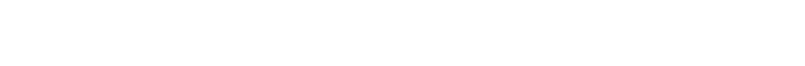
واللوغارتمية المزدوجة والنصف لوغارتمية والتربيعية بالنبة للفئة الحيازية الاوله ذات للسعة الحيوانية القل من

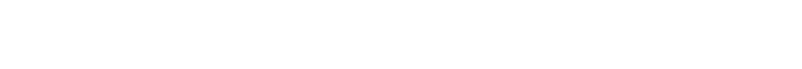

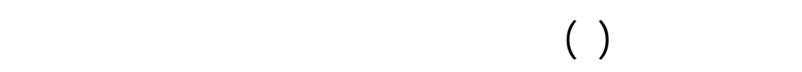
النماذجسواء من وجهه الظرر الاقتصاية او الاحصائية

وهوموضح بالمعادلة التالية:

$$
\begin{aligned}
& \text { ص= } \\
& \left.{ }^{* *}(0, \mu q) \quad{ }^{* *}(\mu, \mu r) \quad{ }^{* *}(\varepsilon, \mu\urcorner\right) \quad{ }^{* *}(\varepsilon, r \cdot) \\
& \text { ف = = } \\
& \cdot, \Lambda 0=r- \\
& \text { حيث: }
\end{aligned}
$$

ص= الطاقة الانتلجية القنيرية للحوم الحمراء بالطن سا = عددالوحدات الحيولنية سع= كمية الاعلاف المركزة باطن سه = كميةالاعلاف الخضراء بالطن ولستنادَ المي هذا النموذج يتضح ان اهم المتغيرات تاثيراً

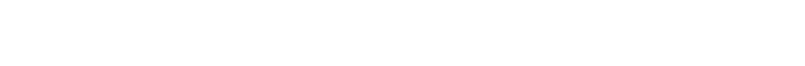

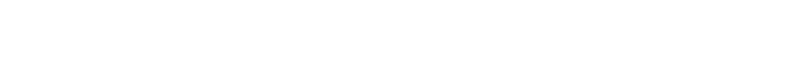
حيوانية هـ:(1) عددالوحدات الحيوانية (س))، (r) مقدار الاعلاف المركزة مقدرة بالطنساع) (ب) مقدار الاعلاف الخضراء مقدرة بالطن (سه).

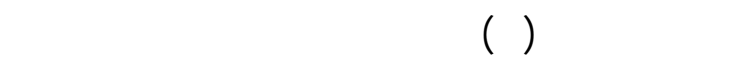

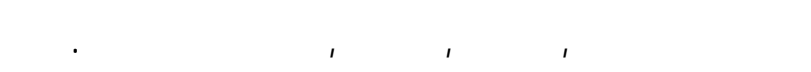

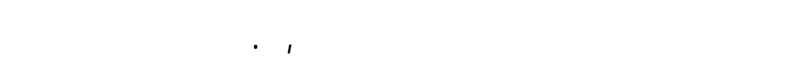
التحديد المعل(ر-r-r) حواله 10, · مما يعنف ان المتغيرات 


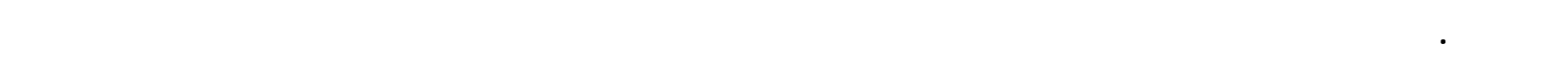

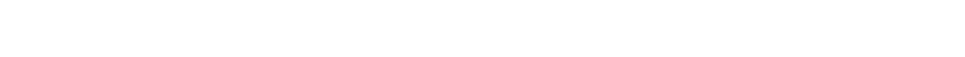

\begin{tabular}{|c|c|c|}
\hline ف & P- & الصور الرباdنية \\
\hline${ }^{* *}|0 \varepsilon, \mu|$ & ·, 10 & 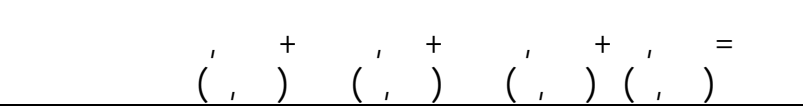 \\
\hline${ }^{* *} \mathrm{T0}, \mathrm{VV}$ & $\cdot, \mathrm{Vr}$ & 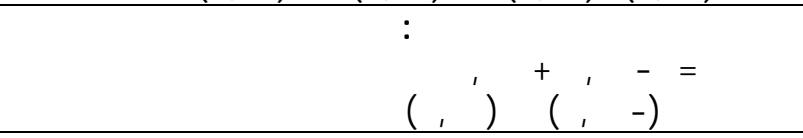 \\
\hline${ }^{* *} \mid \| r, \mathrm{~V}$ & $\cdot 70$ & 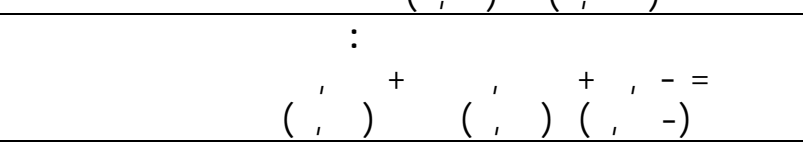 \\
\hline (*, & - 0 - & 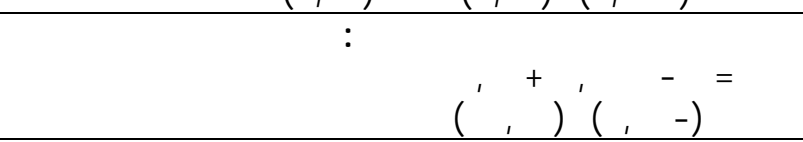 \\
\hline TrV & $\cdot, 07$ & 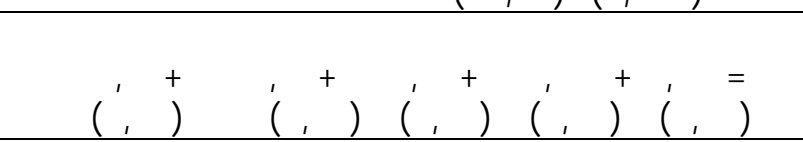 \\
\hline
\end{tabular}

جلط V. الجدارة الفتصادية في الستخدالم الموارد الزراعية في مزالع الفئة الحيازبة الاول بمزالع العينة البحثية في

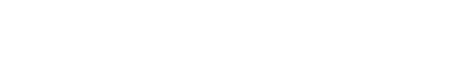

\begin{tabular}{|c|c|c|c|c|c|c|c|}
\hline 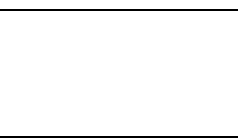 & البكلة الفرصصة بالجنية & قالحمة بالجنية التج & الالنالج & الوحة & المتوصط & المتغير & الرباضنية \\
\hline $1, \mathrm{VV}$ & 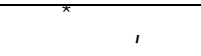 & 797 &., 97 & وحتة حيوانية & $\bar{r}$, & & 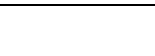 \\
\hline r. & ro. & $0 \cdot v, 0$ & $\because, \mathrm{V}$ & طَن & & سع ( ع & الطية \\
\hline 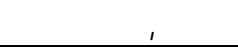 & $r .$. & rq & $\cdot, \cdots \varepsilon$ & طن - طن & $\Lambda, \mathrm{VO}$ & س0. & \\
\hline
\end{tabular}

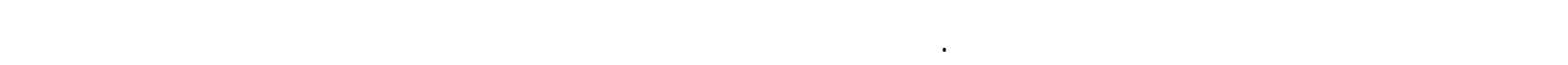

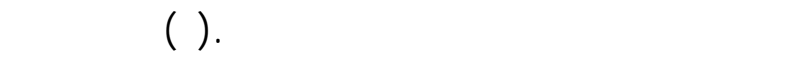

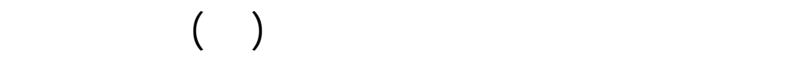

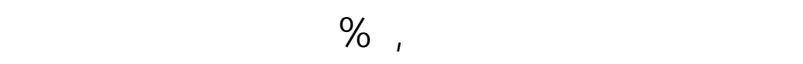

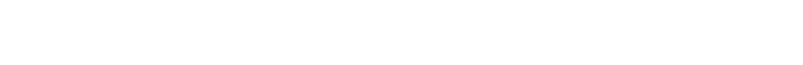

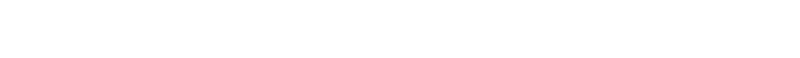
النتلجيتها الحدية مع تكلفة فرصتها البيلة.

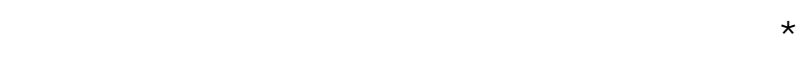

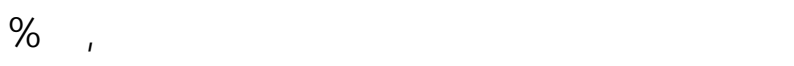
في العلم وان مدة التسمن للوحدات الحيولنية تببلغ 7 الشهور

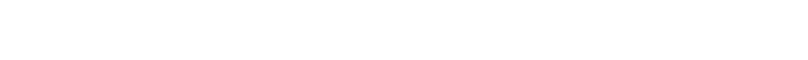

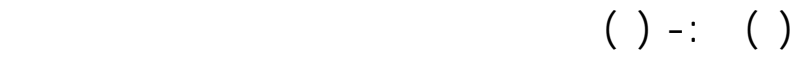
البيلة لمتغير عدد الوحدات الحيولنية(1) تببلغ حواله فيله

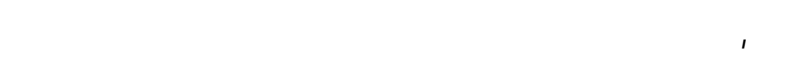

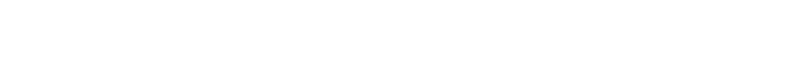
التتاج اللحوم بزياة عدد الوحدات الحيوانية المستغلة حق القياه

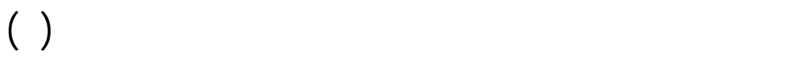
فنبة فتط. قيمة الانتلجية الحية للاعلاف المركزة (سع) الي تكلفة

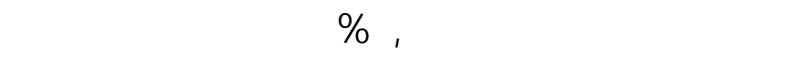
اللحوم يسرفون في لمتخدلم الاعلاف المركزة ويجب الفي 
ص= الطالة الانتلجية الققيرية للحوم الحمراء بالطن

$$
\text { سا = عددالوحدات الحيوانية }
$$

سr= العمالة المزرعية مقدرة برجل / يوم

سع= كميةالاعلاف المركزة بالطن

ولستنادَ الي هذا النموذج يتضح لن اهم المتغيرات

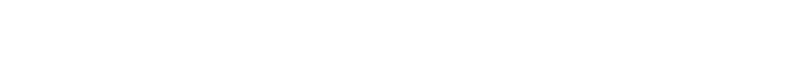

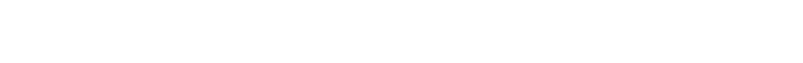

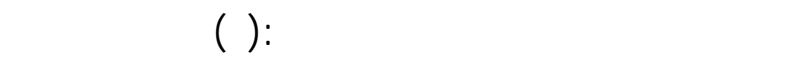

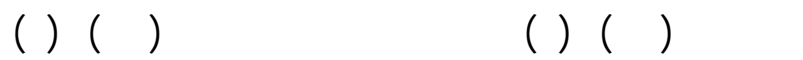
كمية الاعلاف المركزة مقدرة بالطن (سع).

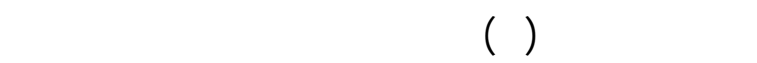

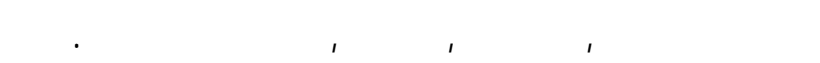

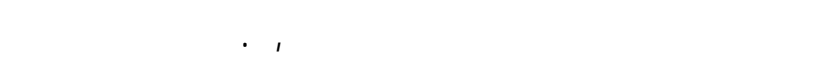

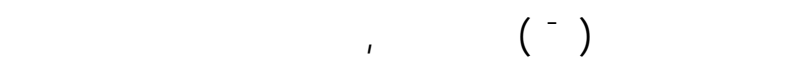

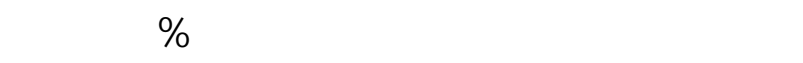
في لجمالى النانج المزرعي من اللحوم في مزارع الفئة الفئة

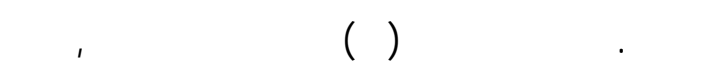

$7 \times 11, r 0 \times v \cdots$

تكلفة الفرصة لبيلة $\operatorname{Ir} \times 1 \cdots$

ثلنيا: - الدلات الانتلجية الحوم الحمراء في مزراع المئة الحيازبة الثلنية: -

بلستخدلم تحليل الانحدار المرحلى المتعدد لققير الدالات الانتلجية لمزارع النتاج للحوم الحمراء في الصورة الطنية

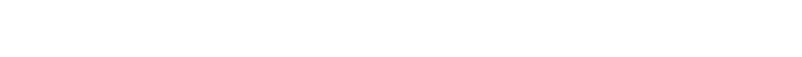
بالنبة للفئة الحيازية الاوله ذات اللسعة الحيولنية لكثر من

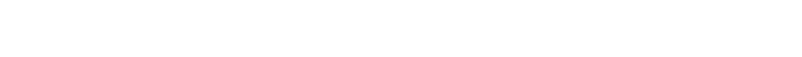

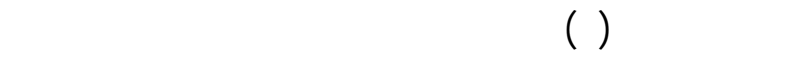
النماذجسواء من وجهه الظر الاقتصاية او الاحصائية وهوموضح بالمعادلة التالية:

$$
\begin{aligned}
& \text { ص= 00, • + + } \\
& \left.{ }^{* *}(\varepsilon, r V) \quad{ }^{* *}\left(\mu, \varepsilon{ }^{\mu}\right) \quad{ }^{* *}(I r, 70) \quad *(r, \Lambda\urcorner\right)
\end{aligned}
$$

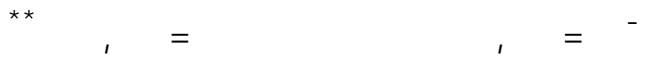

\begin{tabular}{|c|c|c|}
\hline ف & J & الصور الربالضية \\
\hline${ }^{* *} \mid \Psi 0,7$ & $\cdot, \mathrm{V} 7$ & 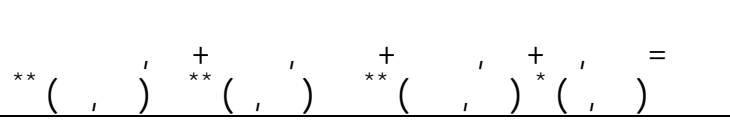 \\
\hline & - 07 & 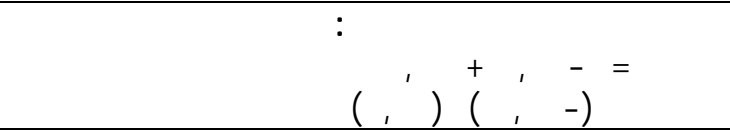 \\
\hline${ }^{* *} \mathrm{TV}, \varepsilon$. & זד, & 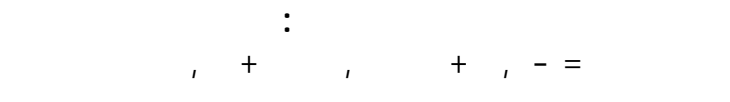 \\
\hline${ }^{* *} \mathrm{~V}, \mathrm{rq}$ & $\cdot, \mathrm{OV}$ & 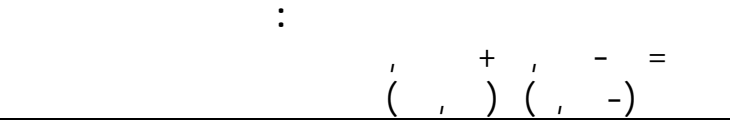 \\
\hline " & $\cdot, 0 \varepsilon$ & 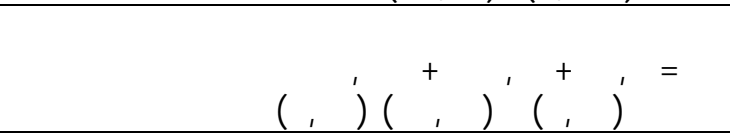 \\
\hline
\end{tabular}

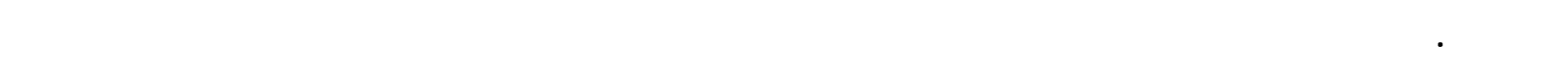

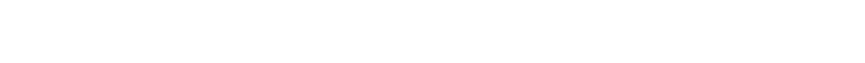

المصدر: جمعت و مُست من بيانات العينة البحثية. 
النتائج المتحصل عليها والوارة بالجدول رقم (9) ان: -

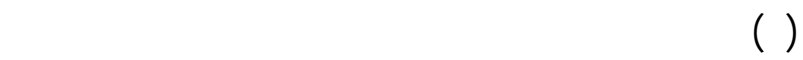

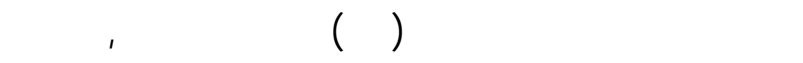
يعنى فوق قيمة التتجيتها الحدية على تكلفة فرصتها البيلة

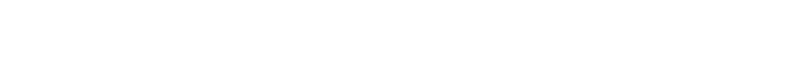

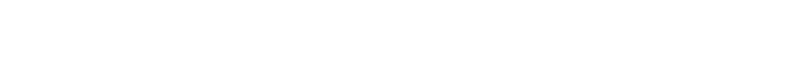

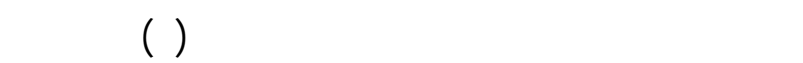

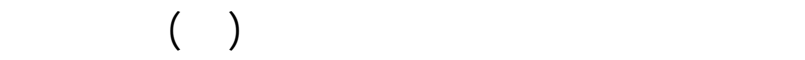

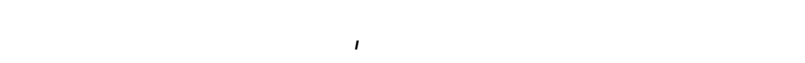
التتلجيتها الحية على تكلفة فرصتها البيلة الى ان المنتجين

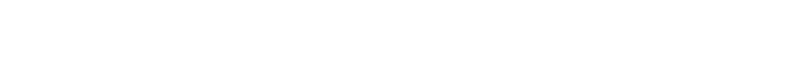
حتى تتساوى قيمة انتلجيتها الحية مع تكلفة فرصنتها البيلة.

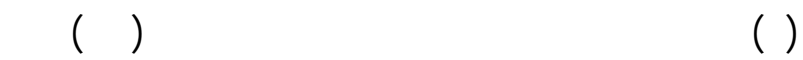

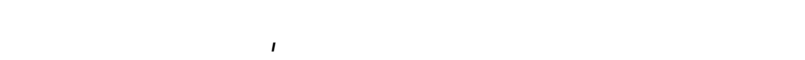

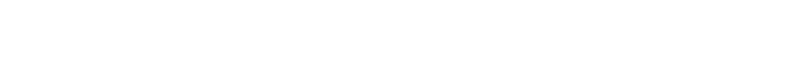
المتتجين يمكنهم زيادة اربلحهم من النتاج اللحوم بزياة المقادير المستخمة من الاعلاف المركزة حقى تتساوى قيمة انتلجيتها الحية مع تكلفة فرصتها البيلة. وبصفة علمة فقد اتضغح من نتائج ققديركل من الانتلجية

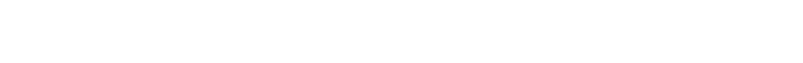
البيلة والوارة بجدوله(11 ، -1) نفوق مزراع الفئة

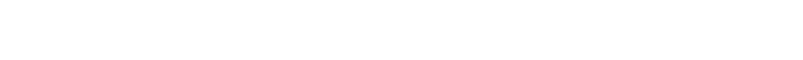
حيث الانتلجية المتوسطة اومن حيث مدي كفاءة لستخدلم الموارد الاقتصادية المزرعية المتلحة، وهو الامر الذي الماني

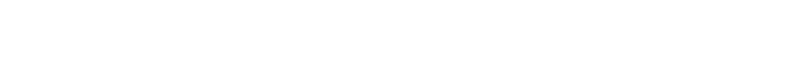

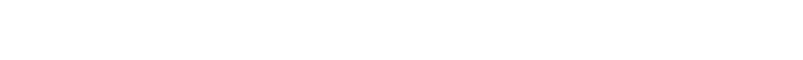
المزرعية الحيولنية.
مما يعنف معنوية تاثير المتغيرات الفسيرية في النموذج

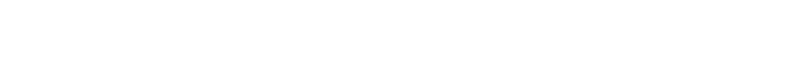
اللحوك الحمراء في العينة البحثية عند المستوى الاحتم اله اله $. \cdot, 1$

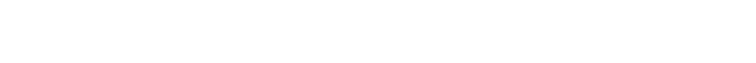
الانتلجيةسالفة النكر يتضح ان الانتلجية الحدية للمتغيرات

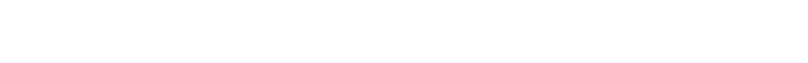

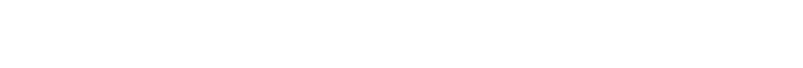

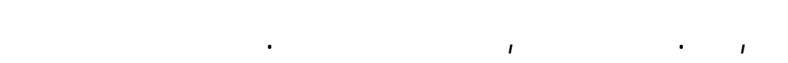

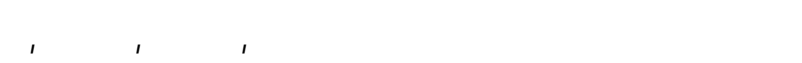

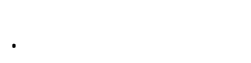
وبمسلب المرونة الانتلجية للمتغير(س) التضح انها تبلغ

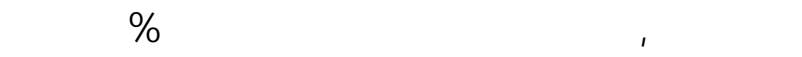

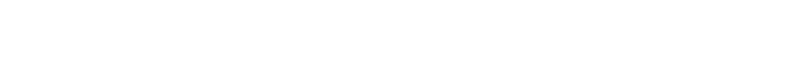
الاتجله مقداره ع,"\% في لجمالى النانج المزرعى من اللحوم. وبالنسة للمرونة الانتلجية للمتغير (سr) فقد بلغت

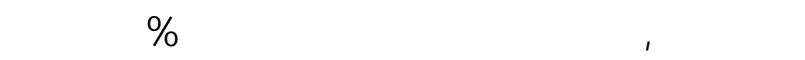
العمالة المزرعية يترتب عليه تغيراً في نشس الاتجه مقداره

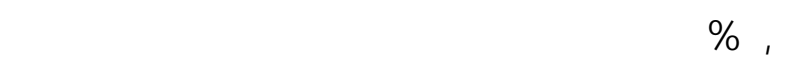

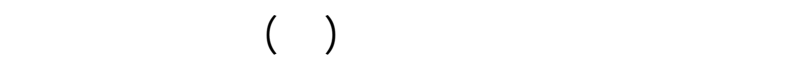

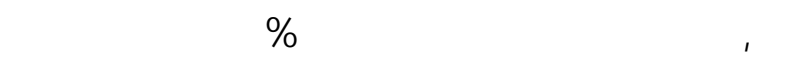

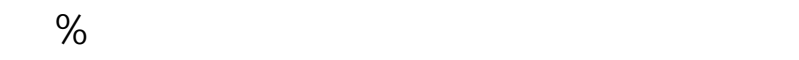
لجمالى النانج المزرعى من اللحوم الحمراء بمزارع الفئة فئة الثانية بعينة الدرلسة.

وقيلس الكفاءة الافتصاية للموارد المزرعية المستغلة

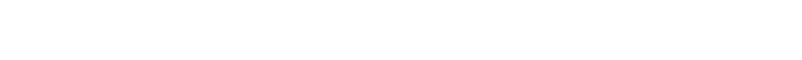
بعينة الدرلسة وذك بنقدير نسبة قيمة الانتلجية الحية لهذه الموارد الل تكلفة فرصتها البيلة للتعرف على الجدارة الجدارة الاقتصادية في لمستخدم المواردسالفة الذكر في ظل سنيادة

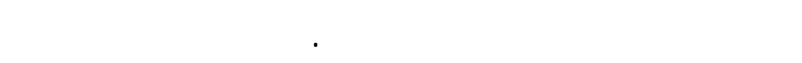


جطل 9. الجدارة لافتصالية في الستخدل الموارد الزراعية في مزالرع الفئة الحيازبة الثلنية بمزالع العينة البحثية في

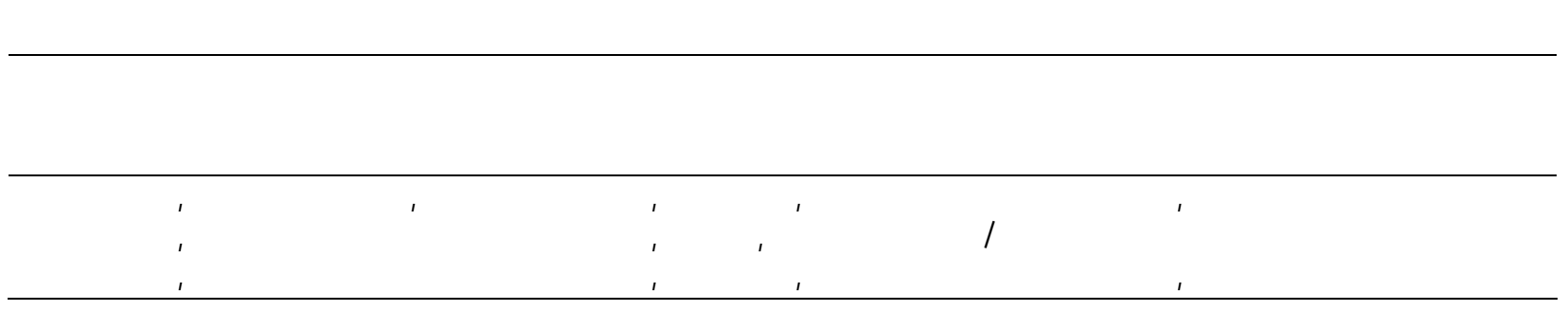

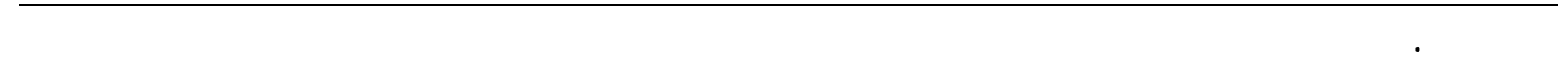

الجسية

\begin{tabular}{|c|c|c|}
\hline \multicolumn{2}{|c|}{ الانتلجية المتوططة المواردد الانتلجية بالالن } & \multirow{2}{*}{ الموارد الانناجية } \\
\hline النغئة الثانية & الفئة لالول & \\
\hline$\cdot, \boldsymbol{\mu} 19$ & $\cdot, 197$ & الوحدات الحيولنية (س) \\
\hline$\cdot, \cdot r$ & - & العمالة المزرعية () \\
\hline ع & rr & الاعلاف المركزز \\
\hline- & س & الاعلاف الخضراء (سo) \\
\hline
\end{tabular}

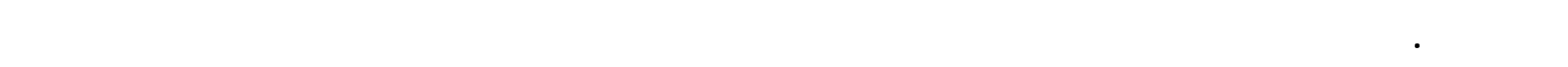
البحية بلفليم النواربة بلارالضي الجية فية الجية الجية

\begin{tabular}{|c|c|c|}
\hline \multicolumn{2}{|c|}{ لاننتلجية المتووطة لاموارد الانتلجية بالالن } & \multirow{2}{*}{ الموارد الانتلجية } \\
\hline أخئة الثانية & الفئة الأل & \\
\hline$\Lambda, \mu \varepsilon$ & $\mathrm{V}, \mathrm{rA}$ & الوحدات الحيولنية (س) \\
\hline$\varepsilon, \vee\urcorner$ & - & العمالة المز \\
\hline $1, \cdot 9$ & · & الاعلاف الـ \\
\hline - & $\cdot$ • & الاعلاف الخضراء (سo) \\
\hline
\end{tabular}

المصدر: جمعت ومُسبت من من جدوله رقم V V

\section{الم ـراججع}

لحمد الفيل ولخرون، قيلس وتحليل الكفاءة الانتلجية والتسويقية

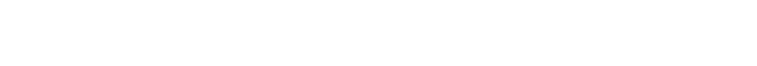

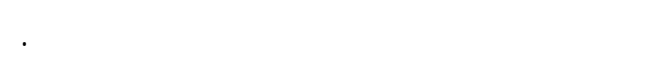

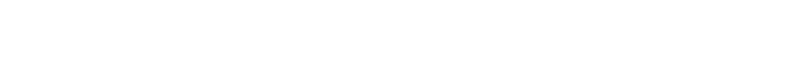

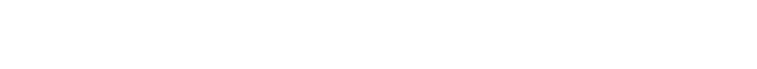
مجلة الإكندرية للبحوث الزراعية، مجلد טيسمبر 1994. الحسين عبدالطيف الصيفي، تحلل إقتصادي للمقتصد الزراعي

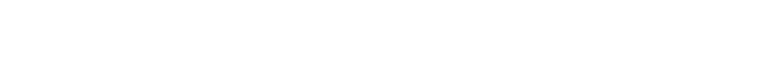
كتورة، قمم الإقتصاد الزراعي، كلية الزراعة، جلمعة

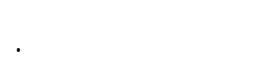

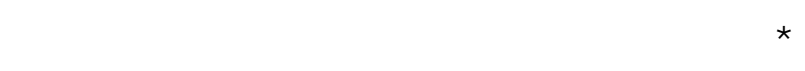
حواله ....... جنية وثمن الوحة الحيوانية يبلغ حواله

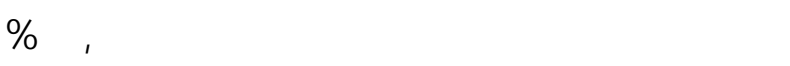

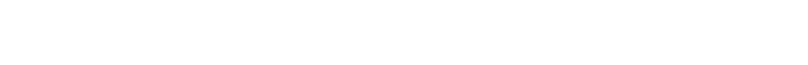
فتط.

$$
7 \times 11, r o \times v \cdots
$$

تكلفة الفرصة البديلة= $\operatorname{Ir} \times 1 \cdots$ 
محمد محمد الخولانى ولخرون، أثر سيلسات الإصلاح

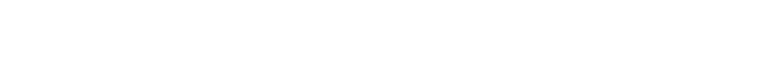

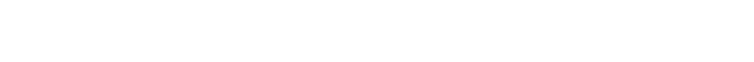

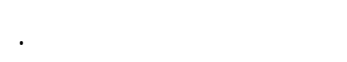

مديرية الزراعة بالنوبارية، سجلات الانتاج الحيوانف ،بيلنت غيرمنشورة، 1999.

منية بهاء الدن هسن على، إقتصاسيت لمتيراد اللحوم الحمراء

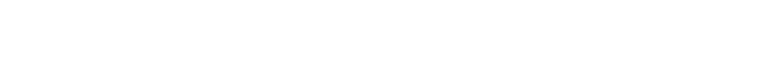

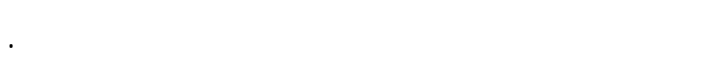

Head ,E.O and Dillon, J.L, Agriculture Production Function, Iowa Stata University Press, Ames, U. S. A ,1966.
الهسين عبد الطيف الصيفي ومحمدمحمد الملهى، درلمة تحليلة

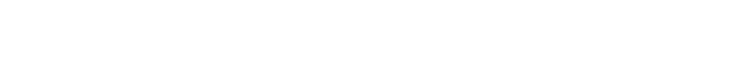

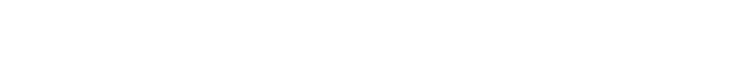

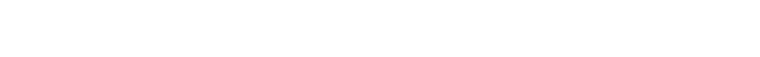

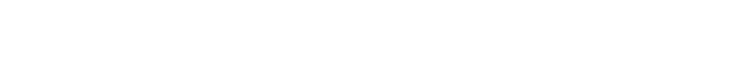

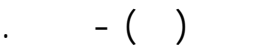
محمد عبدالخاق دعبس، الطلب على اللحوم في مصر، رسالة ملجستير، قم الإقتصاد الزراعي، كلية الزراعة، جلمعة عني شهن، 1999.

\title{
SUMMARY \\ The Economic Efficiency of the Red Meat Production Farms in New Lands (Case Study in Nuberia Region)
}

\author{
Amr Abd El-Hamied Refat
}

The main objectives of the study were to estimate the production function of red meat production farms in Nubaria region,to determine the main factors affect the dimension and degree oh using the agricultural economic resources efficiently at the red meat production farms in the area under the study.

The study is depend on primary data collected from a sample of 75 red meat production farms in Nuberia district. The sample was divided into tow categories, the first category includes 40 farms that contains 1-5 animal units each, while the second category include 35 farms of more than 5 animal units the statistical analysis used include steo-wise multiregression as well as some other models such as linear,logarithm,semi-long and quadratic for both categories under study.

The results of the study has shown that the most important variables thet affect the red meat production farms category are; number of animal units $\left(\mathrm{x}_{1}\right)$, quantity of concentrated forage per ton $\left(\mathrm{x}_{4}\right)$, and, quantity of green feed per $\operatorname{ton}\left(\mathrm{x}_{5}\right)$, the production elasticity estimated to these variables were $0.49,0.32$ and 0.063 respectively. the estimated ratio of MPV to opportunity cost of these resources in order to identify economic efficiency of the utilization of the above use both concentrated and the green feed,and to receive equivalent values of MPV and opportunity cost,it is recommended that must reduce the quantity used of the both feed.

The result of the study has shown that the most important variables that affect the red meat production farms of the second category are; ;number of animal units $\left(\mathrm{x}_{1}\right)$, quantity of concentrated forage per ton $\left(\mathrm{x}_{4}\right)$, and ,quantity of green feed per ton $\left(\mathrm{x}_{5}\right)$, the production elasticity estimated to these variables were $0.34,0.23$ 0.20 respectively. the estimated ratio of MPV to opportunity cost of these resources in order to determine economic efficiency of the utilization of the above mentioned resources were about 2.03, 0.67 and 0.27 respectively,which means the MPV was better than the opportunity cost of these three variables. The producers can increase their profit from the meat production through increasing the animal units and quantity used from both concentrated and green forage, in order to get equivalent values of MPV and opportunity cost.

In general, it has been shown that the secomd farm category was better than the frist category with respect to both the average productivity and efficiency of the utilization of the agricultural economic and the increase available which means the increment in the average productivity and the increase in the agricultural economic resources efficiency of the animal farm used will increase as the farm animal capacity increase. 